Keywords: HER2 testing; HercepTest; endometrial carcinoma; prognostic biomarkers; chromogenic in situ hybridisation; metastatic biopsies; antibody-drugs conjugate; trastuzumab

\title{
HER2 expression patterns in paired primary and metastatic endometrial cancer lesions
}

Mari Kyllesø Halle 1,2, Ingvild Løberg Tangen 1,2, Hege Fredriksen Berg ${ }^{1,2}$, Erling Andre Hoivik ${ }^{1,2}$, Karen K Mauland ${ }^{1,2}$, Kanthida Kusonmano ${ }^{3,4}$, Anna Berg ${ }^{1,2}$, Antoni Hurtado 5,6, Karl Henning Kalland ${ }^{2,7}$, Anne M Øyan ${ }^{7,8}$, Ingunn Stefansson 9,10 , Olav K Vintermyr ${ }^{9,10}$, Henrica M Werner ${ }^{1,2}$, Ingfrid S Haldorsen ${ }^{11,12}$, Jone Trovik ${ }^{1,2}$, Helga B Salvesen ${ }^{1,2, *}$ and Camilla Krakstad ${ }^{\star, 1,2}$

${ }^{1}$ Department of Obstetrics and Gynaecology, Haukeland University Hospital, Jonas Lies vei 72, Bergen 5053, Norway; ${ }^{2}$ Centre for Cancer Biomarkers, Department of Clinical Science, University of Bergen, Jonas Lies vei 72, Bergen 5053, Norway; ${ }^{3}$ Computational Biology Unit, University of Bergen, Nygårdsgaten, Bergen 5008, Norway; ${ }^{4}$ Bioinformatics and Systems Biology Program, School of Bioresources and Technology, King Mongkut's University of Technology Thonburi, Bangkhuntien, Bangkok, 10150 Thailand; ${ }^{5}$ Breast Cancer Research group, Nordic EMBL Partnership, Centre for Molecular Medicine Norway (NCMM), University of Oslo, P:O: 1137 Blindern, Oslo 0318, Norway; ${ }^{6}$ Department of Genetics, Institute for Cancer Research, the Norwegian Radium Hospital, Montebello, 0310 Oslo, Norway; ${ }^{7}$ Department of Microbiology, Haukeland University Hospital, Jonas Lies vei 65, Bergen 5021, Norway; ${ }^{8}$ Department of Clinical Science, University of Bergen, Jonas Lies vei 65, Bergen 5021, Norway; ${ }^{9}$ Department of Pathology, Haukeland University Hospital, Jonas Lies vei 65, Bergen 5021, Norway; ${ }^{10}$ The Gade Laboratory of Pathology, Department of Clinical Medicine, University of Bergen, Jonas Lies vei 65, Bergen 5021, Norway; ${ }^{11}$ Department of Radiology, Haukeland University Hospital, Jonas Lies vei 65, Bergen 5021, Norway and ${ }^{12}$ Section of Radiology, Department of Clinical Medicine, University of Bergen, Jonas Lies vei 65, 5021 Bergen, Norway

Background: Despite successful implementation of drugs targeting the human epidermal growth factor receptor 2 (HER2) receptor in breast and gastric cancers, the potential of HER2 as a therapeutic target in other cancers has been less studied, including endometrial cancer. We investigated expression levels of HER2 (ERBB2) in a large cohort of endometrial cancer lesions, also including complex atypical hyperplasia and metastatic lesions.

Methods: 67 precursor lesions, 790 primary endometrial cancers and 383 metastatic lesions were investigated for HER2 expression in relation to clinicopathologic features and outcome. Protein levels were assessed by immunohistochemistry (using the HercepTest and staining index (SI) criteria), mRNA levels by microarrays and amplification status by chromogenic in situ hybridisation.

Results: High HER2 protein levels were significantly associated with features of aggressive disease and increased mRNA ERBB2 levels. HER2 expression defined by the SI proved to be a better predictor of survival compared with the HercepTest. A discordant HER2 expression pattern between paired primary and metastatic lesions was detected, revealing substantial reduction in HER2 expression from primary to metastatic disease.

Conclusions: Loss of HER2 expression is common in metastatic endometrial cancer lesions and assessment of HER2 levels in the metastatic lesions may be important to define the potential benefit of anti-HER2 treatments in endometrial cancer patients.

*Correspondence: Professor C Krakstad; E-mail: camilla.krakstad@uib.no

Deceased.

Received 4 July 2017; revised 26 October 2017; accepted 26 October 2017; published online 23 November 2017

(C) 2018 Cancer Research UK. All rights reserved 0007-0920/18 
Endometrial cancer is the most frequent gynaecologic malignancy in industrialised countries (Torre et al, 2015), yet the progress of implementing tailored treatment for women with advanced and recurrent disease is slow. The primary treatment for endometrial cancer patients is hysterectomy and bilateral salpingoophorectomy. Thus, adjuvant treatment is directed toward any remaining metastatic tissue. Chemotherapy is currently the standard treatment for systemic recurrent/metastatic endometrial cancer, yet the response rates are low and overall survival is short (Fleming, 2015). In the era of individualised cancer therapy, biomarkers for treatment of metastatic disease are needed.

Proteins expressed on the surface of tumour cells can be selectively targeted, and one of the most frequently targeted proteins is the human epidermal growth factor receptor 2 (HER2), a well-characterised receptor anchored to the cell membrane coded by the gene ERBB2. Amplifications in ERBB2 have been linked to response to HER2-targeting agents such as the monoclonal antibodies trastuzumab and lapatinib, which has revolutionised treatment of HER2-positive breast cancer (Ahmed et al, 2015). HER2-inhibiting compounds are currently being tested in a broad range of cancer types. Case report studies have confirmed clinical activity of trastuzumab in recurrent endometrial carcinoma in vivo (Jewell et al, 2006; Villella et al, 2006; Santin et al, 2008), yet trastuzumab as single agent showed no significant benefit in a phase II clinical trial with HER2-overexpressing tumours (Fleming et al, 2010). Lack of proper pre-selection of patients with ERBB2 amplification and/or HER2 overexpression could partly explain the poor clinical activity observed in HER2-directed trials with endometrial cancer patients (Santin, 2010; Leslie et al, 2012). Strategies combining trastuzumab with other agents are under development, and may prove to be more efficient than single-agent trastuzumab in endometrial cancer treatment. Endometrial serous carcinomas, a biological aggressive subtype of endometrial cancer, are known to exhibit high levels of ERBB2 amplification (17-47\%, (Slomovitz et al, 2004; Santin et al, 2005; Cancer Genome Atlas Research N et al, 2013; Growdon et al, 2015; Jones et al, 2015)) and HER2 overexpression (14-80\% (Buza et al, 2014; Jones et al, 2015)). High rates of resistance to conventional therapy are reported for the serous cancers, and the development of new treatment strategies is therefore important.

In breast cancer, both retrospective and prospective studies indicate a substantial discrepancy in HER2 status between primary and recurrent cancer lesions (Amir et al, 2012; Vignot et al, 2012), and recently the assessment of HER2 status in recurrent/metastatic breast cancer lesions has been added to Advanced Breast Cancer consensus guidelines (Cardoso et al, 2017). HER2 status in primary endometrial cancer tissue is well characterised, yet so far, relationships between HER2 status in primary and associated metastatic lesions are not documented. The aim of this study was to investigate HER2 status in a large prospective series of complex atypical hyperplasia $(\mathrm{CAH})$, primary tumours and metastatic lesions including endometrioid, serous, carcinosarcomas, clear cell and undifferentiated carcinomas. To evaluate the relevance of HER2 as a target for patients with metastatic or recurrent endometrial cancer, HER2 expression in metastatic lesions was characterised and compared with corresponding primary tumour lesions. To our knowledge, this is the first comprehensive study describing HER2 status in paired primary and metastatic endometrial cancers.

\section{MATERIALS AND METHODS}

Ethics statement. This study was approved by the Norwegian legislation and research permission and was granted by the Norwegian Social Data Service (15501), the Western Regional
Committee for Medical and Health Research Ethics and regional Institutional Review Board (IRB 2009-2315). Participants gave informed written consent.

Patient samples. A population-based patient series including 790 patients diagnosed with primary endometrial cancer were prospectively collected in a population based setting in Hordaland County (Norway) from 2001 to 2015. Patient age, stage of disease, histologic type, histologic grade, recurrence status, DNA ploidy status, myometrial infiltration, treatment and follow-up were obtained from the clinical records. The median follow-up for survivors was 48 months (range $0-147$ ). A series of 67 endometrial CAH was prospectively collected from 2001-2012. Clinical information was collected as for the endometrial cancer patients, yet no follow-up data were available from patients with $\mathrm{CAH}$. Oestrogen receptor (ER) and progesterone receptor (PR) was stained and scored as previously described (Engelsen et al, 2008a; Krakstad et al, 2012; Tangen et al, 2014). Formalin-fixed paraffinembedded (FFPE) tissue from hysterectomy specimens and metastatic biopsies were assessed and mounted in TMAs as previously described (Engelsen et al, 2008b). FFPE tissue from metastatic biopsies was available from 192 patients (in total 383 lesions). All patients were staged according to the current International Federation of Gynecology and Obstetrics staging system (Pecorelli, 2009). The TMA method has previously been described and validated in several studies (Kononen et al, 1998; Hoos et al, 2001; Stefansson et al, 2004). In brief, areas with the highest tumour grade and purity were selected on haematoxylin and eosin-stained slides. Tissue cylinders $(0.6 \mathrm{~mm})$ (three from primary tumours and $\mathrm{CAH}$ and one to three from metastatic lesions) were punched from selected areas and mounted into a recipient paraffin block using a custom-made precision instrument (Beecher instruments, Silver Spring, MD, USA).

Immunohistochemical staining of HER2. Immunohistochemical (IHC) staining of HER2 was performed on TMA sections $(5 \mu \mathrm{m})$, which were dewaxed with xylene, rehydrated in graded ethanol and boiled for $15 \mathrm{~min}$ for antigen retrieval in target retrieval solution ( $\mathrm{pH}$ 9, Dako, Glostrup, Denmark). Sections were blocked for peroxidase activity (S2023, Dako) and incubated for $1 \mathrm{~h}$ at room temperature with polyclonal anti-HER2 antibody (A 0485, Dako) diluted 1:400. Secondary HPR-conjugated antibody was applied for $30 \mathrm{~min}$ in room temperature followed by Diaminobenzidine (K 4010, Dako) for $8 \mathrm{~min}$. Counterstaining with haematoxylin was performed before dehydration and mounting.

Evaluation of staining. The sections were scored according to the HercepTest criteria and in line with the updated recommendations for HER2 assessment in breast cancer (Wolff et al, 2013; Rakha et al, 2015) on a scale from 0 to $3+$ : no staining or membrane staining in $<10 \%(0)$, faint partial staining in $>10 \%(1+)$, weak to moderate $(2+)$ or strong $(3+)$ staining of the entire membrane in $>10 \%$ of the tumour cells. Only membrane staining of the invasive tumour was evaluated, and cytoplasmic staining was considered non-specific and not included in this scoring As negative control, we used normal tissue known not to express HER2, such as connective tissue adjacent to the tumour cells. When 74 cases were scored independently by two individual researchers (MKH and ILT) the inter-observer value was very good (kappa value $=0.89$ ) for the HercepTest Score using two categories $(3+v s<3+)$ and good (kappa value=0.74) using four categories. HercepTest Score $2+$ was defined as moderate and $3+$ as high HER2 expression. When evaluating multiple metastatic lesions from the same patient with divergent HercepTest Score, the lesion(s) with the highest HercepTest Score was selected for comparison.

Simultaneously, all TMA sections were evaluated by the semiquantitative, staining index (SI), where both intensity of the 
staining and portion of positive tumour cells were considered. The SI is a well-established scoring method (Engelsen et al, 2008a) that has previously been used for several biomarkers and in several tumour types (Aas et al, 1996; Bachmann et al, 2006; Lin et al, 2008). Staining intensity was classified from 0 (no staining) to 3 (strong staining). Quantity of stained tumour cells was classified as $0,1(<10 \%), 2(10$ to $50 \%)$ and $3(>50 \%)$. SI was defined as the product of intensity and staining area as previously described. The inter-observer agreement was good (kappa $=0.79)$ using two categories (0-6 vs 9) and within all categories (kappa=0.68) within the 74 cases scored individually by two researchers (M.K.H and I.L.T).

In addition, HER2 levels assessed by the HercepTest, were reevaluated in relation to apical staining patterns in 764 primary tumours. The tumours were classified according to presence of apical HER2 membrane staining as either 'apical HER2 loss' or apical loss 'not detected'. To evaluate if apical loss affected the prognostic value of the HercepTest, tumours with strong lateral/ basolateral staining and apical loss were reclassified as $3+$ tumours. However, the classical HercepTest criteria were followed in all subsequent analyses.

Chromogenic in situ hybridisation. ERBB2 gene amplification status was analysed on TMA sections $(5 \mu \mathrm{m})$ using chromogenic in situ hybridisation (CISH). This is an established method in breast cancer treatment for detecting amplified ERBB2. Included in the analysis were 169 metastatic lesions with overlapping IHC results. The CISH assays were run at the Department of Pathology, University of Bergen following the manufacturer's protocol (BenchMark Ultra, Ventana Medical Systems, Tucson, Arizona). The CISH evaluations were performed blinded and without knowledge of HER2 scoring from IHC. For each cancer lesion, visible gene probe signals were counted in 20 nuclei by standard light microscopy, and mean ;gene probe signals per cell were calculated and defined as absolute gene copy number. In line with the American Society of Clinical Oncology (ASCO)/College of American Pathologists (CAP) guideline recommendations normal copy number was defined as an absolute ERBB2 copy number $<4$, borderline amplification as $4-6$ and amplified ERBB2 as six gene copies or more (Wolff et al, 2013).

Gene expression analyses. Fresh tissue for RNA extraction was available for 184 of the primary tumours. RNA was extracted using the RNeasy Mini Kit (Qiagen, Hilden, Germany) followed by hybridisation onto Agilent Whole Human Genome Microarrays 44 k (Cat. No G4112F). Signal intensities were defined using the software J-express (Molmine, Bergen, Norway) (www.molmine.com) (Dysvik and Jonassen, 2001), and median spot intensity was used to determine intensity of signals. The data set included expression values for genes represented by 41001 probes and expression data were quantile normalised.

Statistical analysis. The data were analysed by the software package SPSS (Statistical Package of Social Science) version 24 (IBM, Armonk, NY, USA). Weighted kappa was used to assess inter-observer agreement value when categories were ordered. All $P$-values are two-sided and considered statistically significant if $<0.05$. Associations between groups were analysed with Person $\chi^{2}$, linear-by-linear-association or Fisher's exact test for categorical variables, and the Mann-Whitney $U$-test for continuous variables as appropriate. Univariate survival analysis of death due to endometrial cancer (disease-specific survival; DSS) was performed using the Kaplan-Meier (product-limit) method, and differences in survival between groups were calculated by the log-rank test (Mantel-Cox).

\section{RESULTS}

High HER2 expression associates with aggressive disease and poor survival in primary endometrial cancer. HER2 expression was detected by IHC and evaluated by the HercepTest and the SI criteria. For evaluation using the HercepTest criteria, FDA guidelines were followed and only membranous staining was considered (Supplementary Figure 1). High HER2, defined as HercepTest Score $3+$ was significantly associated with established markers of aggressive disease (Table 1) and poor outcome (Figure 1A). The 5-year DSS for patients with primary tumours with low HER2 expression $(0-2+)$ was 87 , vs $77 \%$ for patients with HER2-high expressing tumours $(3+)(P=0.006$, Figure $1 \mathrm{~A})$. mRNA levels of ERBB2 was positively correlated to protein levels of HER2 measured by the HercepTest criteria in 184 overlapping samples $(P=0.01$, Figure 1B). A gradual increase in number of HER2 high tumours was observed according to aggressiveness of histologic type and grade (Figure $1 \mathrm{C}, P<0.001$, trend test). The highest proportion of high HER2 $(3+)$ was found among serous tumours $(49 \%)$, followed by clear celled $(30 \%)$, carcinosarcomas

\begin{tabular}{|c|c|c|c|}
\hline & HER2 H & cepTest & \\
\hline & $\begin{array}{c}\text { Low } \\
(0-2+)\end{array}$ & $\begin{array}{l}\text { High } \\
(3+)\end{array}$ & $P$-value ${ }^{b}$ \\
\hline Variables $(n)^{a}$ & $n(\%)$ & $n(\%)$ & \\
\hline $\begin{array}{c}\text { Age }(n=790) \\
\text { Mean age }\end{array}$ & 64 & 70 & $<0.001^{c}$ \\
\hline $\begin{array}{l}\text { FIGO-09 stage }{ }^{d}(n=790) \\
\text { III } \\
\text { II-IV }\end{array}$ & $\begin{array}{l}561(84) \\
95(77)\end{array}$ & $\begin{array}{l}105(16) \\
29(23)\end{array}$ & 0.04 \\
\hline $\begin{array}{l}\text { Histologic type }(n=790) \\
\text { Endometrioid } \\
\text { Serous papillary } \\
\text { Clear cell } \\
\text { Carcinosarcoma } \\
\text { Undifferentiated }\end{array}$ & $\begin{array}{l}561(88) \\
37(51) \\
21(70) \\
26(77) \\
11(85)\end{array}$ & $\begin{array}{l}80(12) \\
35(49) \\
9(30) \\
8(23) \\
2(15)\end{array}$ & $<0.001$ \\
\hline $\begin{array}{l}\text { Histologic grade }{ }^{\mathbf{e}}(n=623) \\
\text { Grade } 1 / 2 \\
\text { Grade } 3\end{array}$ & $\begin{array}{l}458(89) \\
87(79)\end{array}$ & $\begin{array}{l}55(11) \\
23(21)\end{array}$ & 0.003 \\
\hline $\begin{array}{l}\text { Recurrence }(n=789) \\
\text { No recurrence } \\
\text { Metastatic at primary } \\
\text { Later recurrence }\end{array}$ & $\begin{array}{l}518(85) \\
41(75) \\
96(77)\end{array}$ & $\begin{array}{l}92(15) \\
14(25) \\
28(23)\end{array}$ & 0.03 \\
\hline $\begin{array}{l}\text { DNA ploidy }(n=500) \\
\text { Diploid } \\
\text { Aneuploid }\end{array}$ & $\begin{array}{l}335(87) \\
82(73)\end{array}$ & $\begin{array}{l}52(13) \\
31(27)\end{array}$ & $<0.001$ \\
\hline $\begin{array}{l}\text { Myometrial infiltration }(n=788) \\
\quad<50 \% \\
>50 \%\end{array}$ & $\begin{array}{l}403(83) \\
250(82)\end{array}$ & $\begin{array}{l}80(17) \\
54(18)\end{array}$ & 0.66 \\
\hline $\begin{array}{l}\text { ER }(n=649) \\
\text { Positive } \\
\text { Negative }\end{array}$ & $\begin{array}{l}410(85) \\
141(85)\end{array}$ & $\begin{array}{l}73(15) \\
25(15)\end{array}$ & 0.99 \\
\hline $\begin{array}{c}\text { PR }(n=647) \\
\text { Positive } \\
\text { Negative }\end{array}$ & $\begin{array}{l}434(87) \\
115(78) \\
\end{array}$ & $\begin{array}{l}65(13) \\
33(22) \\
\end{array}$ & 0.006 \\
\hline \multicolumn{4}{|c|}{ 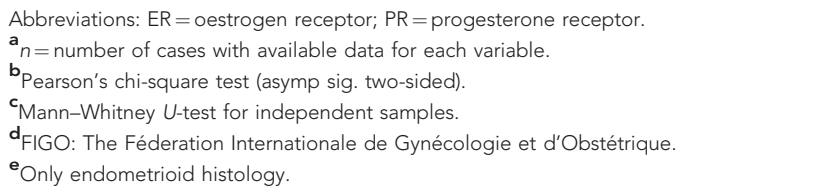 } \\
\hline
\end{tabular}




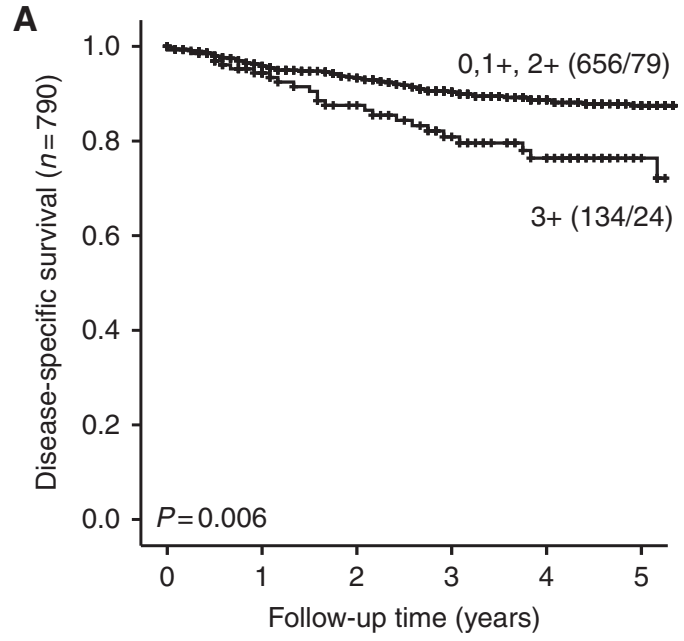

B
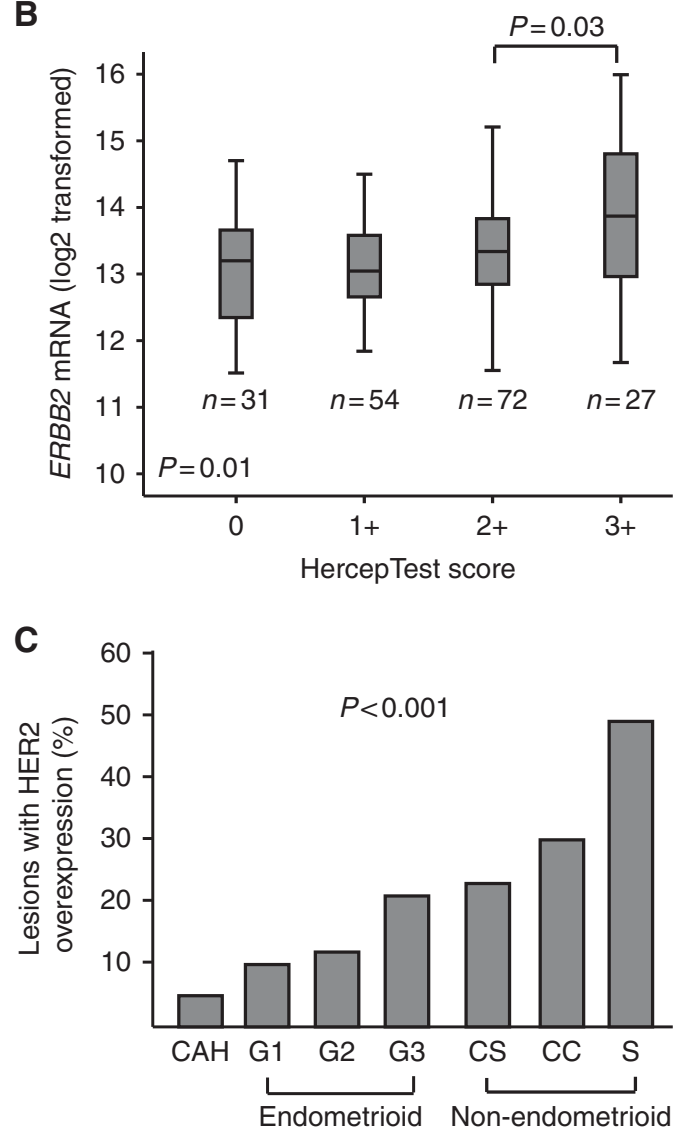

Figure 1. Relationship between HER2 expression (assessed by the HercepTest) and survival, mRNA expression and aggressiveness of disease. Kaplan-Meier curves representing endometrial carcinoma disease-specific survival (DSS) according to HER2 protein levels, assessed by the HercepTest criteria (A). ERBB2 mRNA expression in tumours with increasing HercepTest Score (B). Proportion of HER2 overexpression $(3+)$ with increasing disease aggressiveness, also including complex atypical hyperplasia (C). $\mathrm{CAH}=$ Complex atypical hyperplasia, CC = Clear cell carcinoma, CS = Carcinosarcoma, G1 = Grade 1, G2 = Grade 2, G3 = Grade 3, S = Serous carcinoma.

(23\%), endometrioid grade $3(21 \%)$, grade $2(12 \%)$ and grade 1 (10\%) tumours. Within the investigated $\mathrm{CAH}, 4 \%$ exhibited high HER2 levels (Figure 1C). In multivariate survival analysis, high HER2 (measured by the HercepTest) and ERBB2 mRNA levels were not associated with poor survival when correcting for age, stage and grade (Supplementary Table $1 \mathrm{~A}$ and B, respectively).

To investigate whether the scoring criteria for detecting HER2positive endometrial cancers could be improved, an alternative method, the SI for evaluation of IHC was applied. When the SI criteria were used, cytoplasmic staining was included in the evaluation. Based on observed differences in univariate survival analysis according to the SI criteria, high HER2 was defined as SI 9 and SI 0-6 as HER2 low (Supplementary Figure 2A). High HER2 (SI 9) was significantly associated with markers for aggressive disease (Supplementary Table 2) and survival (Figure 2A). In univariate survival analysis, patients with low HER2 (SI 0-6) tumours had a 5-year DSS of 87 , vs $61 \%$ for patients with high HER2 (SI 9) tumours $(P<0.001$, Figure 2A). A strong positive correlation between the HER2 protein levels assessed by the SI criteria and ERBB2 mRNA expression was observed $(P=0.002$, Supplementary Figure $2 \mathrm{~B}$ ). Considering the strong prognostic impact of this alternative scoring method (SI) compared with the more established HercepTest, we performed survival analyses, using SI criteria, within subgroups of patients with known poor survival and treatment response. High HER2 (SI 9) was associated with poor outcome within ER- and/or PR-negative tumours $(P=0.008$, Supplementary Figure $2 \mathrm{C})$ and within non-endometrioid tumours $(P=0.03$, Supplementary Figure 2D). These patients were not identified using the HercepTest criteria (data not shown). Interestingly, also within HercepTest Score $3+$ tumours, High HER2 SI (SI 9) associated with poor outcome compared with SI $0-6(P=0.003$, Figure $2 \mathrm{~B})$. In multivariate survival analysis corrected for age, stage and grade, High HER2 (SI 9) independently associated with survival, although not significant (HR 1.61 (95\% CI: 0.91-2.85, $P=0.1$ ), Supplementary Table 1C).

Lack of apical HER2 membrane staining resulting in basolateral/ lateral staining patterns has previously been described in serous endometrial carcinomas (Buza et al, 2013). We carefully examined staining patterns to investigate whether this affects the prognostic impact of HER2. Lack of apical staining was observed in $45 \%$ of the primary tumours. Staining patterns are exemplified in Figure 2C (full membrane staining) and Figure 2D (loss of apical staining). A significant difference in apical staining patterns was observed among different histologic groups $(P=0.03$, Supplementary Table 3). The serous tumours (Figure $2 \mathrm{C}$ and $\mathrm{D}$, right panel) had the highest prevalence of apical HER2 loss (64\%) followed by clear cell carcinomas (46\%), endometrioid carcinomas (44\%), undifferentiated tumours (38\%) and carcinosarcomas (33\%) (Supplementary Table 3). Reclassifying tumours with loss of apical HER2 staining and strong lateral/basolateral membranous staining pattern into HercepTest $3+$ Score $(P=0.039$, Figure $2 \mathrm{E})$ did not affect overall survival compared with results from the HercepTest criteria $(P=0.036$, Figure $2 \mathrm{~F})$, indicating that these cases could be scored as $3+$ when evaluating HER2 staining.

Divergent HER2 expression patterns in primary and corresponding metastatic lesions. HER2 expression levels in primary and associated metastatic lesions were compared according to the established HercepTest criteria (Figure $3 \mathrm{~A}$ and $\mathrm{B}, n=142$ ). Of the primary tumours with corresponding metastatic lesions, $11 \%$ (9/81) of the endometrioid tumours and $41 \%$ (25/61) of the nonendometrioid tumours had high HER2 levels $(3+)$. Among the non-endometrioid tumours, $57 \%(35 / 61)$ were serous and in these $57 \%(20 / 35)$ had HER2-high levels $(3+)$ and $31 \%(11 / 35)$ had intermediate HER2 levels $(2+)$ (Figure $3 \mathrm{~B})$.

In the endometrioid paired primary and metastatic cases, a decrease in lesions with high HER2 was found for metastatic sites $(n=7)$ as compared with the primary sites $(n=9)$ (Figure 3A). For non-endometrioid metastatic lesions (Figure $3 \mathrm{~B}$ ), the highest percentage of HER2-high $(3+)$ was found within the serous subgroup, yet only $45 \%$ (9/20) of the patients with HER2-high 

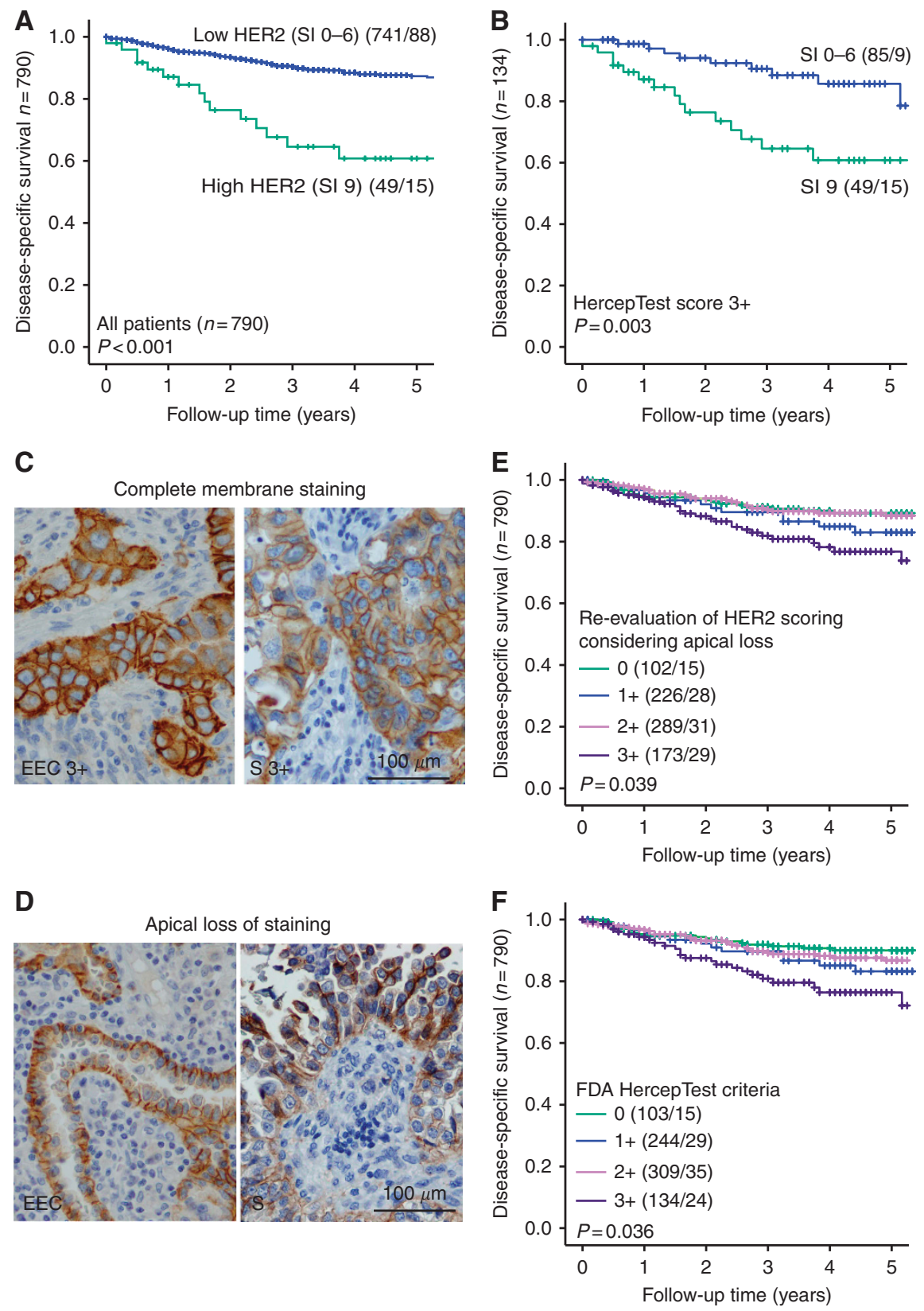

Figure 2. Disease-specific survival (DSS) according to the staining index (SI) criteria and apical HER2 loss. Low HER2 represents SI 0-6 and High HER2 represents SI 9. Kaplan-Meier curves representing endometrial carcinoma DSS according to HER2 protein levels assessed by the SI criteria (A). DSS according to HER2 assessed by the SI criteria for patients with HercepTest Score $3+$ tumours (B). Tumours with complete HER2 membrane staining (C) and HER2 apical loss (D) in endometrioid (EEC; left) and serous (S; right) tumours. DSS according to the HercepTest after re-evaluation of HER2 scoring considering apical loss in four groups $(E)$. DSS according to the original FDA HercepTest criteria in four groups (F).

primary tumours retained high HER2 levels in the metastatic setting. Among the serous primary tumours with low HER2 (0$2+)(n=15)$, three patients had HER2-high metastatic lesions. For patients with carcinosarcoma, the number of HER2-high tumours increased from three to five from the primary to the metastatic setting. In clear cell carcinomas, a small fraction of HER2-high lesions was found both in primary and metastatic lesions. In undifferentiated tumours, one primary and no metastatic lesions were HER2-high (Figure 3B). Altogether, high HER2 levels were found in $18 \%(25 / 142)$ of the metastases investigated. Within the HER2-high primary tumours, 62\% (21/34) had HER2-low corresponding metastatic lesions; and within the HER2-low primary tumours, only 11\% (12/108) had HER2-high corresponding metastases (Table 2). Overall, discordant expression of HER2 (HercepTest $0-2+v s 3+$ ) between primary and corresponding metastatic lesion(s) was observed in 23\% (33/142) of the patients, with similar rates for patients treated with chemoand/or radiation therapy between primary and recurrent disease (data not shown). Interestingly, within cases with available multiple metastatic lesions, 16\% (10/62) had divergent HER2 expression levels $(0-2+v s 3+)$ within the different metastatic sites evaluated (Figure 3 ).

For patients with few treatment options, expression of HER2 may open for targeted therapy also when expression levels are lower than HercepTest Score 3+. If a HercepTest Score $2+$ is found to be sufficient for effective HER2-directed treatment in 
A

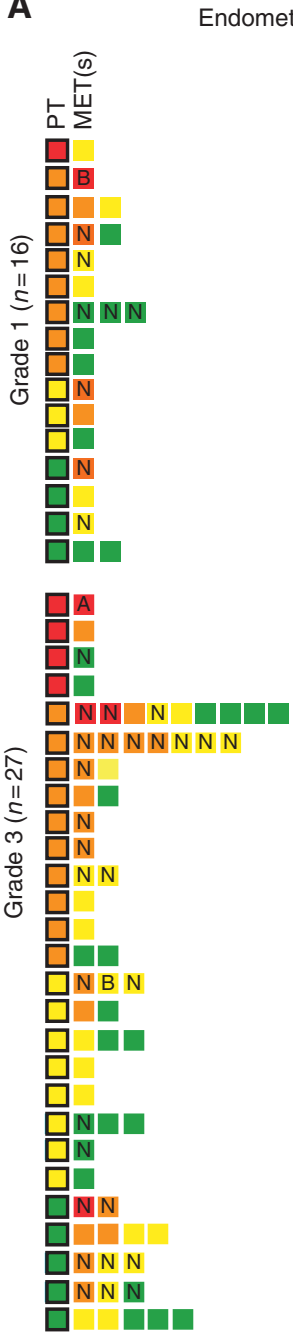

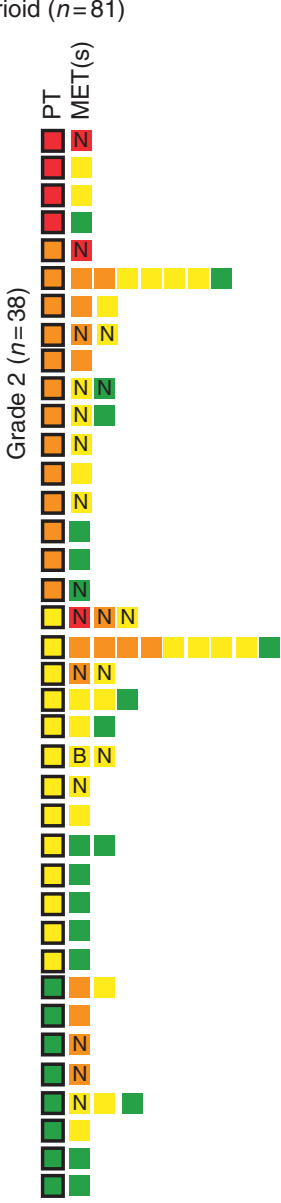

B

Non-endometrioid $(n=61)$
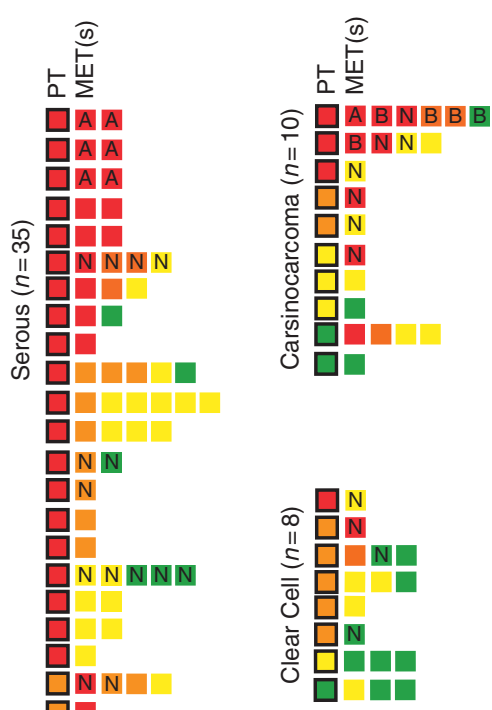

品

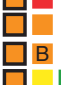

$\square \mathrm{N}$

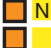

虽
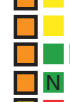

$\square \mathrm{N}$

$\square$ $\square \square \square$

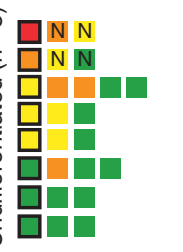

ERBB2 copy number in metastatic lesions (CISH)

$\mathrm{N}=$ Normal $\quad \mathrm{B}=$ Borderline $\quad \mathrm{A}=$ Amplified

Figure 3. Expression patterns in HER2 metastatic lesions compared with the primary tumour. HER2 expression of the individual metastatic lesion compared with its expression in in primary tumour in endometrioid (A) and non-endometrioid $(\mathbf{B})$ cases. MET(s) $=$ Metastasis or Metastases, $\mathrm{PT}=$ primary tumour, Undiff $=$ Undifferentiated tumour.

Table 2. Comparison of HER2 status assessed by the HercepTest criteria in 142 primary and matched metastatic sites

\begin{tabular}{|c|c|c|c|c|c|}
\hline & \multicolumn{5}{|c|}{ HercepTest score in metastatic sites } \\
\hline & 0 & $1+$ & $2+$ & $3+$ & Total \\
\hline & $n(\%)$ & $n(\%)$ & $n(\%)$ & $n(\%)$ & $n(\%)$ \\
\hline \multicolumn{6}{|c|}{ HercepTest score in primary sites } \\
\hline 0 & $8(35)$ & $8(35)$ & $5(22)$ & $2(8)$ & $23(16)$ \\
\hline $1+$ & $17(49)$ & $10(29)$ & $5(14)$ & $3(8)$ & $35(24)$ \\
\hline $2+$ & $15(29)$ & $21(40)$ & $9(17)$ & $7(14)$ & $50(36)$ \\
\hline $3+$ & $4(12)$ & $12(35)$ & $5(15)$ & $13(38)$ & $34(24)$ \\
\hline Total & $44(31)$ & $51(35)$ & $24(17)$ & 25 (17) & $142(100)$ \\
\hline
\end{tabular}

metastatic endometrial cancer, 40 additional patients (28\%) included in this cohort could potentially benefit from such treatment regime, $65(36 \%)$ patients in total.

The HercepTest scoring criteria sensitively predict ERBB2 amplification in metastatic endometrial cancer lesions. Relation between amplification status and protein expression were investigated in a subset of the metastatic lesions $(n=169)$. A positive correlation between increasing ERBB2 copy number and HER2 protein levels obtained from the HercepTest $(P<0.001$, Figure $4 \mathrm{~A})$ and the SI criteria $(P=0.005$, Figure $4 \mathrm{~B})$ was found.

To test which of the two IHC scoring methods could predict ERBB2 amplification status most accurately, the specificity and sensitivity of the two methods in predicting amplification status of $E R B B 2$ was calculated. Borderline amplification was not considered in the sensitivity and specificity calculations. A HercepTest Score $3+$ identified 10 of 11 (91\%) lesions found to be amplified by CISH, whereas SI 9 only detected 4 of 11 amplified lesions (36\%) (Supplementary Table 4A, Figure 4C). However, among lesions with normal copy number by CISH, 18 of 144 (12\%) scored $3+$ and only 1 of $144(0.7 \%)$ scored SI 9\% (Supplementary Table 4A, Figure 4D). Based on these numbers, the HercepTest criteria had a sensitivity of $91 \%$ and a specificity of $88 \%$ in predicting amplification status of ERBB2 in metastatic lesions, whereas the sensitivity and specificity of SI scoring method were $36 \%$ and 99.3\%, respectively (Supplementary Table 4B). The positive and negative predictive value of the HercepTest was 36 and 99\%, and for SI 80 and 96\%, respectively (Supplementary Table 4B). Although many false positives, the HercepTest criteria are thus a better predictor of ERBB2 amplification status than the alternative method SI. 

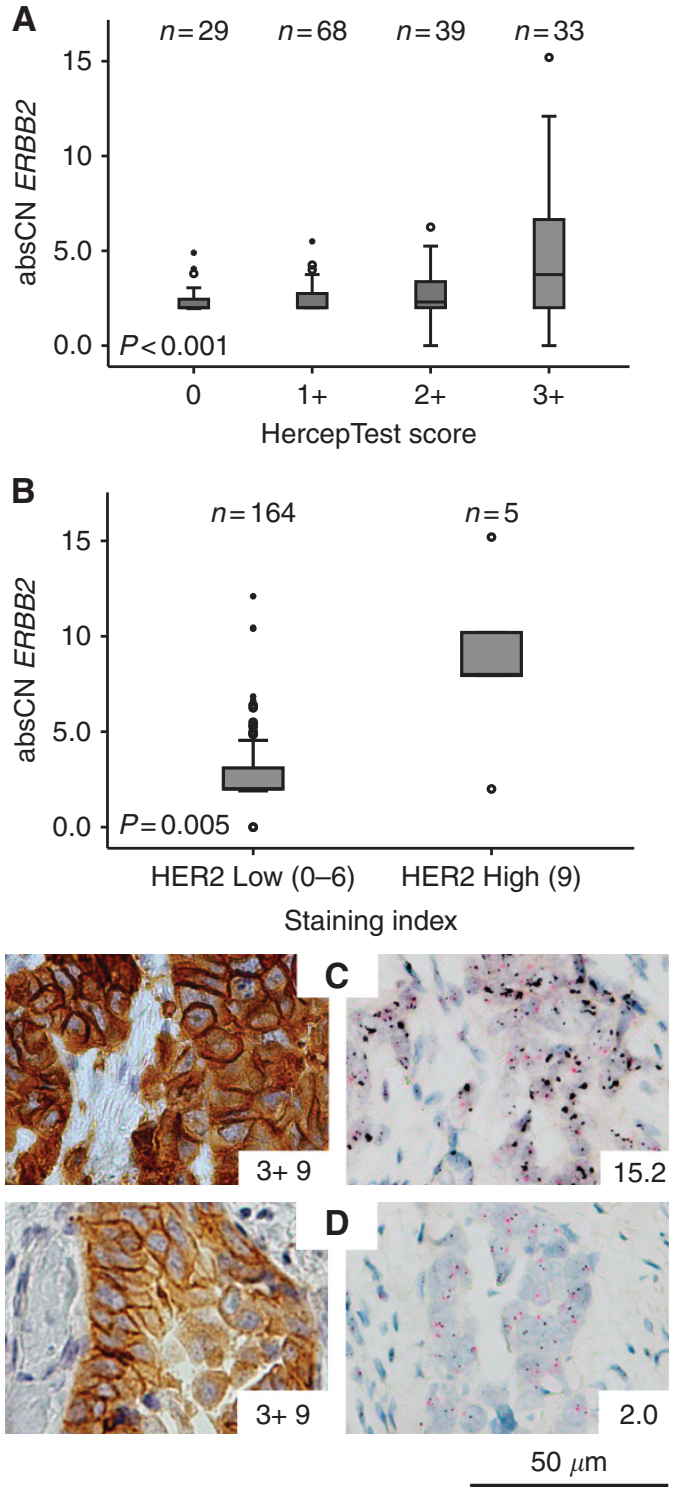

Figure 4. Comparison of HER2 levels assessed by immunohistochemistry (HercepTest and staining index) and absolute copy number (absCN) detected by chromogenic in situ hybridisation. $\mathrm{AbsCN}$ in tumours with increasing HercepTest Score (A) and Staining Index (B) in metastatic lesions. Metastatic lesion with HER2 overexpression (HercepTest $3+/$ SI 9) and amplified ERBB2 (absCN of 15.2) (C) or no amplification in ERBB2 (abs 2.0) (D).

\section{DISCUSSION}

HER2 activation initiate signal transduction through the mitogenactivated protein kinase and PI3K signalling leading to transcription of genes that promote oncogenic transformation via cell survival, proliferation, angiogenesis and metastasis (Yarden and Sliwkowski, 2001). Amplification in ERBB2, the gene coding for HER2, is a genomic alteration associated with high-risk endometrial carcinomas (Cancer Genome Atlas Research N et al, 2013; Salvesen et al, 2009, 2012; Jones et al, 2017), and distinct subsets of high-risk endometrial cancer patients could be potential candidates for HER2-directed treatment. To the best of our knowledge, this is the largest population-based endometrial cancer cohort investigating HER2 expression. We find high HER2 $(3+)$ in $16 \%$ of primary tumours. A considerable variability in reported HER2 expression rates exists (Buza et al, 2014). This is at least partly owing to variability in testing methods, interpretation, and scoring criteria applied. Contrasting breast and gastric cancer, no established HER2 testing guidelines exist in endometrial cancer, and refined and more standardised testing protocols for each cancer type are needed to identify patients that may benefit from HER2-directed treatment. To deal with HER2-testing challenges, an alternative scoring method, the SI was evaluated. Patients with very poor survival were identified using high HER2 (SI 9), even in subgroups of ER and/or PR negative, non-endometrioid and HercepTest $3+$ tumours. Although fewer patients were identified with SI 9 than with HercepTest Score 3+, this alternative scoring method yields as a strong prognostic marker in HER2-high patients. When reevaluating the primary tumours included on TMAs we observed some degree of loss of apical HER 2 staining in $45 \%$ of the tumours. Within different histologic types, significant differences in the prevalence of this pattern were detected, and loss of apical staining was most often observed in serous tumours (64\%) in line with previous findings (Buza et al, 2013). When allowing tumours with strong lateral/basolateral staining and apical HER2 loss for a $3+$ score, no effect was seen on survival, indicating that also these patients should be included in the $3+$ group in guidelines for HER2 evaluation in endometrial cancer. The present study explores HER2 expression using TMAs, and not full sections. The TMA method enables high-throughput, cost and time effective analysis and reduces the amount of tissue used as well as potential batch effects during staining. However, selected areas may not be representative of the entire tumour. Although TMA is a very useful tool in a research setting, optimal cutoff values for markers explored by IHC, FISH or CISH should be validated using full sections before clinical implementation.

The current study cohort included 641 endometrioid, 72 serous and 30 clear cell carcinomas, 34 carcinosarcomas and 13 undifferentiated tumours. Distinct differences in HER2 expression between the histologic types were discovered. Serous carcinomas, clear cell carcinomas, carcinosarcomas and high-grade endometrioid carcinomas had the highest proportions of tumours exhibiting high HER2 $(3+)$ (in 49\%, 30\%, 23\% and $21 \%$, respectively), contrasting low-grade endometrioid tumours, with only $11 \%$. This is in line with previous studies in endometrial cancer, and supportive of our finding that HER2 is associated with aggressive disease (Slomovitz et al, 2004; Morrison et al, 2006; Jones et al, 2017). Patients with non-endometrioid and high-grade endometrioid tumours are in need of improved and more targeted therapeutic strategies, and our study confirms that HER2-directed treatment could represent a potential option for subsets of these patients.

Systemic treatment in endometrial cancer is predominantly aimed towards metastatic and recurrent disease. Hence, knowledge of metastatic HER2 levels is essential to identify endometrial cancer patients suitable for HER2-directed treatment. This is to date the largest characterisation of HER2 expression in metastatic endometrial cancer. Comparable to what is found in breast cancer (Niikura et al, 2012; Criscitiello et al, 2014; Shachar et al, 2017), we find a heterogeneous HER2 expression pattern between primary and corresponding metastatic lesion(s). Our results, based on IHC assessment of HER2, show that conversion from HER2-high $(3+)$ HER2-low $(0-2+)$ status is higher than the opposite conversion (62\% vs $11 \%$, respectively). Especially, within the HER2-high-rich clear cell and serous primary tumours, HER2 overexpression decreased from primary to metastatic setting. If selection of patients is based on HER2 expression in primary tumours, this could partly explain the modest response to HER2-targeted treatment reported in the NCT00006089/GOG181B clinical trial with endometrial cancer patients (Fleming et al, 2010). Although technical issues, including poor reproducibility of the staining and scoring of HER2 may explain some of the observed divergence between primary and corresponding metastatic lesions, tumour 
heterogeneity can be another part of the explanation. Recent studies based on next-generation sequencing of endometrial cancer tissue (Gibson et al, 2016) supports the hypothesis of tumour heterogeneity and sub clonal genetic tumour evolution may lead to variation in, for example, HER2 status in primary and metastatic sites. In line with this, our study has revealed discordant HER2 expression between different metastases obtained from the same patients. This finding suggests that sampling biopsies from multiple metastatic sites could be beneficial to optimise individualised treatment.

Several HER2 resistance mechanisms has been proposed, such as mutated PIK3CA, PTEN loss or increased AKT and S6K phosphorylation in breast cancer treatment (Nagata et al, 2004; Berns et al, 2007; Kataoka et al, 2010), expression of truncated p95HER2 variant, as found in high-grade endometrial tumours (Growdon et al, 2015; Carvajal-Hausdorf et al, 2017) and activation of alternative pathways including other HER family members (Sergina et al, 2007; Pandya et al, 2011). These findings, in addition to our discovery that HER2 expression levels decreases in the metastases may explain some of the challenges with primary trastuzumab resistance found in endometrial cancer (Diver et al, 2015). Altogether, this knowledge should motivate the set-up of clinical trials combining HER2-inhibitors with other agents such as PI3K or mTOR inhibitors affecting downstream parts of the signalling pathway, and the use of, for example, lapatinib or afatinib. Suggested approaches to overcome HER2 resistance are mainly unexplored (Lheureux and Oza, 2016), and combination therapy-targeting cancer cells simultaneously at multiple checkpoints may prove successful in treatment of HER2-positive endometrial cancer.

Amplifications in ERBB2 have been linked to the effects of HER2-targeting agents such as the monoclonal antibodies trastuzumab, pertuzumab and lapatinib in breast cancer (Loibl and Gianni, 2016). We find a fair level of concordance between HER2 IHC results and ERBB2 CISH results. Yet a comparison showed that many metastatic lesions express high HER2 protein levels $(3+)$ without amplification. The HercepTest criteria were more sensitive (99\%) in detecting ERBB2 amplifications than the SI criteria. Still, a specificity of $88 \%$ yields a high level of false positives, and to ensure valid assessment of ERBB2 amplification status in metastatic endometrial cancer, CISH or fluorescent in situ hybridisation (FISH) should be preferred.

In serous endometrial carcinomas, the ERBB2 amplification status has been found to be heterogeneous (Buza and Hui, 2013) and the ASCO/CAP criteria ( $>30 \%$ cell stained) have been found to correspond better with FISH as compared with the HercepTest ( $>10 \%$ cell stained) (Buza et al, 2013). Combined with our findings this could imply that the ideal cutoff for stained cells could lie $\sim 30 \%$ in endometrial cancer, at least for serous tumours. To improve reliability of HER2 scoring in endometrial cancer, both scoring method and staining patterns should be further investigated in future studies.

True ERBB2 amplified tumours are believed to be HER2 driven, and could be potential targets for monoclonal HER2-targeting antibodies. Recently, antibody-conjugated drugs (ADCs) have shown promising results even when HER2 is not a tumour driver. The ADC, trastuzumab emtansine (Genentech/Roche) delivers the cytotoxic agent emtansine into HER2 expressing cells (Junttila et al, 2011) and has shown effect in recurrent or metastatic HER2positive serous endometrial cancer (Santin et al, 2017). SYD985 (Synthon Biopharmaceuticals BV) show high efficacy in uterine serous carcinoma mouse xenograft models with both strong $(3+)$ and low to intermediate (that is, $1+/ 2+$ ) HER2 expression (Black et al, 2016). These results suggest inclusion of endometrial cancer patients with metastatic tumours with HercepTest Score $\geqslant 2+$ regardless of $E R B B 2$ amplification status. In the present study, the HercepTest Score $\geqslant 2+$ in metastatic serous, clear cell and carcinosarcomas were $51 \%, 31 \%$ and $29 \%$, respectively. These patients have poor prognosis, are typically poor responders to conventional therapy (English et al, 2013) and could be candidates for HER2-directed ADCs.

A recent study on HER2 in combination with the promising prognostic biomarker L1CAM in early endometrioid tumours revealed that HER2-expressing cells further aggravated the a priori poor prognosis of the L1CAM-positive endometrioid tumours. Furthermore, double immunostaining revealed a mutually exclusive staining pattern for HER2 and L1CAM on the level of tumour cells (Abdel Azim et al, 2017). IHC detection of both these two surface proteins could serve as a diagnostic tool to select patients with potential benefit from HER2- and/or L1CAM targeting therapies.

In conclusion, our data confirm that the loss of HER2-positive status in metastatic lesions may occur in patients with HER2positive primary tumours. Tissue sampling of metastatic lesions may be helpful in optimising therapeutic agents according to the molecular phenotype of the metastatic disease rather than that of the primary tumour. We have identified potential subgroups for HER2-directed treatment of metastatic or recurrent endometrial cancer, and based on the high level of HER2-positivity when also including $2+$ tumours in metastatic lesions we encourage ADCbased anti-HER2 approaches in future clinical trials. Still, selection of potential candidates needs to be based on refined HER2 testing protocols and preferably testing in multiple full-section metastatic lesions.

\section{ACKNOWLEDGEMENTS}

We thank Britt Edvardsen, Ellen Valen, Kadri Madissoo, Reidun Kopperud, Tormund Njølstad, Hoang My Hua and Bendik Nordanger for technical assistance. Helse Vest Research Fund, University of Bergen, The Research Council of Norway, The Norwegian Cancer Society (Harald Andersens Legat) and Bergen Research Foundation supported this study.

\section{CONFLICT OF INTEREST}

The authors declare no conflict of interest.

\section{REFERENCES}

Aas T, Borresen AL, Geisler S, SmithSorensen B, Johnsen H, Varhaug JE, Akslen LA, Lonning PE (1996) Specific P53 mutations are associated with de novo resistance to doxorubicin in breast cancer patients. Nat Med 2(7): 811-814.

Abdel Azim S, Sprung S, Mutz-Dehbalaie I, Fessler S, Zeimet AG, Marth C (2017) L1CAM and HER2 expression in early endometrioid uterine cancer. Int J Gynecol Pathol 36(4): 356-363.

Ahmed S, Sami A, Xiang J (2015) HER2-directed therapy: current treatment options for HER2-positive breast cancer. Breast Cancer 22(2): 101-116.

Amir E, Clemons M, Purdie CA, Miller N, Quinlan P, Geddie W, Coleman RE, Freedman OC, Jordan LB, Thompson AM (2012) Tissue confirmation of disease recurrence in breast cancer patients: pooled analysis of multi-centre, multi-disciplinary prospective studies. Cancer Treat Rev 38(6): 708-714.

Bachmann IM, Halvorsen OJ, Collett K, Stefansson IM, Straume O, Haukaas SA, Salvesen HB, Otte AP, Akslen LA (2006) EZH2 expression is associated with high proliferation rate and aggressive tumor subgroups in cutaneous melanoma and cancers of the endometrium, prostate, and breast. J Clin Oncol 24(2): 268-273.

Berns K, Horlings HM, Hennessy BT, Madiredjo M, Hijmans EM, Beelen K, Linn SC, Gonzalez-Angulo AM, Stemke-Hale K, Hauptmann M, Beijersbergen RL, Mills GB, van de Vijver MJ, Bernards R (2007) A functional genetic approach identifies the PI3K pathway as a major 
determinant of trastuzumab resistance in breast cancer. Cancer Cell 12(4): 395-402.

Black J, Menderes G, Bellone S, Schwab CL, Bonazzoli E, Ferrari F, Predolini F, De Haydu C, Cocco E, Buza N, Hui P, Wong S, Lopez S, Ratner E, Silasi DA, Azodi M, Litkouhi B, Schwartz PE, Goedings P, Beusker PH, van der Lee MM, Timmers CM, Dokter WH, Santin AD (2016) SYD985, a novel duocarmycin-based HER2-targeting antibodydrug conjugate, shows antitumor activity in uterine serous carcinoma with HER2/Neu expression. Mol Cancer Ther 15(8): 1900-1909.

Buza N, English DP, Santin AD, Hui P (2013) Toward standard HER2 testing of endometrial serous carcinoma: 4-year experience at a large academic center and recommendations for clinical practice. Mod Pathol 26(12): $1605-1612$

Buza N, Hui P (2013) Marked heterogeneity of HER2/NEU gene amplification in endometrial serous carcinoma. Genes Chromosomes Cancer 52(12): 1178-1186.

Buza N, Roque DM, Santin AD (2014) HER2/neu in endometrial cancer: a promising therapeutic target with diagnostic challenges. Arch Pathol Lab Med 138(3): 343-350.

Cancer Genome Atlas Research N, Kandoth C, Schultz N, Cherniack AD, Akbani R, Liu Y, Shen H, Robertson AG, Pashtan I, Shen R, Benz CC, Yau C, Laird PW, Ding L, Zhang W, Mills GB, Kucherlapati R, Mardis ER, Levine DA (2013) Integrated genomic characterization of endometrial carcinoma. Nature 497(7447): 67-73.

Cardoso F, Costa A, Senkus E, Aapro M, Andre F, Barrios CH, Bergh J, Bhattacharyya G, Biganzoli L, Cardoso MJ, Carey L, Corneliussen-James D, Curigliano G, Dieras V, El Saghir N, Eniu A, Fallowfield L, Fenech D, Francis P, Gelmon K, Gennari A, Harbeck N, Hudis C, Kaufman B, Krop I, Mayer M, Meijer H, Mertz S, Ohno S, Pagani O, Papadopoulos E, Peccatori F, Pernault-Llorca F, Piccart MJ, Pierga JY, Rugo H, Shockney L, Sledge G, Swain S, Thomssen C, Tutt A, Vorobiof D, Xu B, Norton L, Winer E (2017) 3rd ESO-ESMO international consensus guidelines for advanced breast cancer (ABC 3). Ann Oncol 28(1): 16-33.

Carvajal-Hausdorf DE, Schalper KA, Bai Y, Black J, Santin AD, Rimm DL (2017) Objective, domain-specific HER2 measurement in uterine and ovarian serous carcinomas and its clinical significance. Gynecol Oncol 145(1): 154-158.

Criscitiello C, Andre F, Thompson AM, De Laurentiis M, Esposito A, Gelao L, Fumagalli L, Locatelli M, Minchella I, Orsi F, Goldhirsch A, Curigliano G (2014) Biopsy confirmation of metastatic sites in breast cancer patients: clinical impact and future perspectives. Breast Cancer Res 16(2): 205.

Diver EJ, Foster R, Rueda BR, Growdon WB (2015) The Therapeutic Challenge of Targeting HER2 in Endometrial Cancer. Oncologist 20(9): $1058-1068$.

Dysvik B, Jonassen I (2001) J-Express: exploring gene expression data using Java. Bioinformatics 17(4): 369-370.

Engelsen IB, Stefansson IM, Akslen LA, Salvesen HB (2008a) GATA3 expression in estrogen receptor alpha-negative endometrial carcinomas identifies aggressive tumors with high proliferation and poor patient survival. Am J Obstet Gynecol 199(5): 543 e1-543 e7.

Engelsen IB, Stefansson IM, Beroukhim R, Sellers WR, Meyerson M, Akslen LA, Salvesen HB (2008b) HER-2/neu expression is associated with high tumor cell proliferation and aggressive phenotype in a population based patient series of endometrial carcinomas. Int J Oncol 32(2): 307-316.

English DP, Roque DM, Santin AD (2013) HER2 expression beyond breast cancer: therapeutic implications for gynecologic malignancies. Mol Diagn Ther 17(2): 85-99.

Fleming GF (2015) Second-line therapy for endometrial cancer: the need for better options. J Clin Oncol 33(31): 3535-3540.

Fleming GF, Sill MW, Darcy KM, McMeekin DS, Thigpen JT, Adler LM, Berek JS, Chapman JA, DiSilvestro PA, Horowitz IR, Fiorica JV (2010) Phase II trial of trastuzumab in women with advanced or recurrent, HER2-positive endometrial carcinoma: a Gynecologic Oncology Group study. Gynecol Oncol 116(1): 15-20.

Gibson WJ, Hoivik EA, Halle MK, Taylor-Weiner A, Cherniack AD, Berg A, Holst F, Zack TI, Werner HM, Staby KM, Rosenberg M, Stefansson IM, Kusonmano K, Chevalier A, Mauland KK, Trovik J, Krakstad C, Giannakis M, Hodis E, Woie K, Bjorge L, Vintermyr OK, Wala JA, Lawrence MS, Getz G, Carter SL, Beroukhim R, Salvesen HB (2016) The genomic landscape and evolution of endometrial carcinoma progression and abdominopelvic metastasis. Nat Genet 48(8): 848-855.

Growdon WB, Groeneweg J, Byron V, DiGloria C, Borger DR, Tambouret R, Foster R, Chenna A, Sperinde J, Winslow J, Rueda BR (2015) HER2 over- expressing high grade endometrial cancer expresses high levels of p95HER2 variant. Gynecol Oncol 137(1): 160-166.

Hoos A, Urist MJ, Stojadinovic A, Mastorides S, Dudas ME, Leung DH, Kuo D, Brennan MF, Lewis JJ, Cordon-Cardo C (2001) Validation of tissue microarrays for immunohistochemical profiling of cancer specimens using the example of human fibroblastic tumors. Am J Pathol 158(4): $1245-1251$.

Jewell E, Secord AA, Brotherton T, Berchuck A (2006) Use of trastuzumab in the treatment of metastatic endometrial cancer. Int J Gynecol Cancer 16(3): 1370-1373.

Jones NL, Xiu J, Chatterjee-Paer S, Buckley de Meritens A, Burke WM, Tergas AI, Wright JD, Hou JY (2017) Distinct molecular landscapes between endometrioid and nonendometrioid uterine carcinomas. Int $J$ Cancer 140(6): 1396-1404.

Jones NL, Xiu J, Reddy SK, Burke WM, Tergas AI, Wright JD, Hou JY (2015) Identification of potential therapeutic targets by molecular profiling of 628 cases of uterine serous carcinoma. Gynecol Oncol 138(3): 620-626.

Junttila TT, Li G, Parsons K, Phillips GL, Sliwkowski MX (2011) Trastuzumab-DM1 (T-DM1) retains all the mechanisms of action of trastuzumab and efficiently inhibits growth of lapatinib insensitive breast cancer. Breast Cancer Res Treat 128(2): 347-356.

Kataoka Y, Mukohara T, Shimada H, Saijo N, Hirai M, Minami H (2010) Association between gain-of-function mutations in PIK3CA and resistance to HER2-targeted agents in HER2-amplified breast cancer cell lines. Ann Oncol 21(2): 255-262.

Kononen J, Bubendorf L, Kallioniemi A, Barlund M, Schraml P, Leighton S, Torhorst J, Mihatsch MJ, Sauter G, Kallioniemi OP (1998) Tissue microarrays for high-throughput molecular profiling of tumor specimens. Nat Med 4(7): 844-847.

Krakstad C, Trovik J, Wik E, Engelsen IB, Werner HM, Birkeland E, Raeder MB, Oyan AM, Stefansson IM, Kalland KH, Akslen LA, Salvesen HB (2012) Loss of GPER identifies new targets for therapy among a subgroup of ERalpha-positive endometrial cancer patients with poor outcome. Br J Cancer 106(10): 1682-1688.

Leslie KK, Sill MW, Lankes HA, Fischer EG, Godwin AK, Gray H, Schilder RJ, Walker JL, Tewari K, Hanjani P, Abulafia O, Rose PG (2012) Lapatinib and potential prognostic value of EGFR mutations in a Gynecologic Oncology Group phase II trial of persistent or recurrent endometrial cancer. Gynecol Oncol 127(2): 345-350.

Lheureux S, Oza AM (2016) Endometrial cancer-targeted therapies myth or reality? Review of current targeted treatments. Eur J Cancer 59: 99-108.

Lin BY, Utleg AG, Gravdal K, White JT, Halvorsen OJ, Lu W, True LD, Vessella R, Lange PH, Nelson PS, Hood L, Kalland KH, Akslen LA (2008) WDR19 expression is increased in prostate cancer compared with normal cells, but low-intensity expression in cancers is associated with shorter time to biochemical failures and local recurrence. Clinical Cancer Res 14(5): 1397-1406.

Loibl S, Gianni L (2016) HER2-positive breast cancer. Lancet 389(10087): 2415-2429.

Morrison C, Zanagnolo V, Ramirez N, Cohn DE, Kelbick N, Copeland L, Maxwell GL, Fowler JM (2006) HER-2 is an independent prognostic factor in endometrial cancer: association with outcome in a large cohort of surgically staged patients. J Clin Oncol 24(15): 2376-2385.

Nagata Y, Lan KH, Zhou X, Tan M, Esteva FJ, Sahin AA, Klos KS, Li P, Monia BP, Nguyen NT, Hortobagyi GN, Hung MC, Yu D (2004) PTEN activation contributes to tumor inhibition by trastuzumab, and loss of PTEN predicts trastuzumab resistance in patients. Cancer Cell 6(2): $117-127$.

Niikura N, Liu J, Hayashi N, Mittendorf EA, Gong Y, Palla SL, Tokuda Y, Gonzalez-Angulo AM, Hortobagyi GN, Ueno NT (2012) Loss of human epidermal growth factor receptor 2 (HER2) expression in metastatic sites of HER2-overexpressing primary breast tumors. J Clin Oncol 30(6): 593-599.

Pandya K, Meeke K, Clementz AG, Rogowski A, Roberts J, Miele L, Albain KS, Osipo C (2011) Targeting both Notch and ErbB-2 signalling pathways is required for prevention of ErbB-2-positive breast tumour recurrence. $\mathrm{Br} J$ Cancer 105(6): 796-806.

Pecorelli S (2009) Revised FIGO staging for carcinoma of the vulva, cervix, and endometrium. Int J Gynaecol Obstet 105(2): 103-104.

Rakha EA, Pinder SE, Bartlett JM, Ibrahim M, Starczynski J, Carder PJ, Provenzano E, Hanby A, Hales S, Lee AH, Ellis IO. National Coordinating Committee for Breast P (2015) Updated UK Recommendations for HER2 assessment in breast cancer. J Clin Pathol 68(2): 93-99. 
Salvesen HB, Carter SL, Mannelqvist M, Dutt A, Getz G, Stefansson IM, Raeder MB, Sos ML, Engelsen IB, Trovik J, Wik E, Greulich H, Bo TH, Jonassen I, Thomas RK, Zander T, Garraway LA, Oyan AM, Sellers WR, Kalland KH, Meyerson M, Akslen LA, Beroukhim R (2009) Integrated genomic profiling of endometrial carcinoma associates aggressive tumors with indicators of PI3 kinase activation. Proc Natl Acad Sci USA 106(12): 4834-4839.

Salvesen HB, Haldorsen IS, Trovik J (2012) Markers for individualised therapy in endometrial carcinoma. Lancet Oncol 13(8): e353-e361.

Santin AD (2010) Letter to the Editor referring to the manuscript entitled: "Phase II trial of trastuzumab in women with advanced or recurrent HERpositive endometrial carcinoma: a Gynecologic Oncology Group study" recently reported by Fleming et al., (Gynecol Oncol., 116;15-20;2010). Gynecol Oncol 118(1): 95-96author reply 96-7.

Santin AD, Bellone S, Buza N, Schwartz PE (2017) Regression of metastatic, radiation/chemotherapy-resistant uterine serous carcinoma overexpressing HER2/neu with trastuzumab emtansine (TDM-1). Gynecol Oncol Rep 19: 10-12.

Santin AD, Bellone S, Roman JJ, McKenney JK, Pecorelli S (2008) Trastuzumab treatment in patients with advanced or recurrent endometrial carcinoma overexpressing HER2/neu. Int J Gynaecol Obstet 102(2): 128-131.

Santin AD, Bellone S, Van Stedum S, Bushen W, Palmieri M, Siegel ER, De Las Casas LE, Roman JJ, Burnett A, Pecorelli S (2005) Amplification of c-erbB2 oncogene: a major prognostic indicator in uterine serous papillary carcinoma. Cancer 104(7): 1391-1397.

Sergina NV, Rausch M, Wang D, Blair J, Hann B, Shokat KM, Moasser MM (2007) Escape from HER-family tyrosine kinase inhibitor therapy by the kinase-inactive HER3. Nature 445(7126): 437-441.

Shachar SS, Mashiach T, Fried G, Drumea K, Shafran N, Muss HB, Bar-Sela G (2017) Biopsy of breast cancer metastases: patient characteristics and survival. BMC cancer 17(1): 7.

Slomovitz BM, Broaddus RR, Burke TW, Sneige N, Soliman PT, Wu W, Sun CC, Munsell MF, Gershenson DM, Lu KH (2004) Her-2/neu overexpression and amplification in uterine papillary serous carcinoma. J Clin Oncol 22(15): 3126-3132.

Stefansson IM, Salvesen HB, Akslen LA (2004) Prognostic impact of alterations in P-cadherin expression and related cell adhesion markers in endometrial cancer. J Clin Oncol 22(7): 1242-1252.

Tangen IL, Werner HM, Berg A, Halle MK, Kusonmano K, Trovik J, Hoivik EA, Mills GB, Krakstad C, Salvesen HB (2014) Loss of progesterone receptor links to high proliferation and increases from primary to metastatic endometrial cancer lesions. Eur J Cancer 50(17): 3003-3010.

Torre LA, Bray F, Siegel RL, Ferlay J, Lortet-Tieulent J, Jemal A (2015) Global cancer statistics, 2012. CA Cancer J Clin 65(2): 87-108.

Vignot S, Besse B, Andre F, Spano JP, Soria JC (2012) Discrepancies between primary tumor and metastasis: a literature review on clinically established biomarkers. Crit Rev Oncol Hematol 84(3): 301-313.

Villella JA, Cohen S, Smith DH, Hibshoosh H, Hershman D (2006) HER-2/ neu overexpression in uterine papillary serous cancers and its possible therapeutic implications. Int J Gynecol Cancer 16(5): 1897-1902.

Wolff AC, Hammond ME, Hicks DG, Dowsett M, McShane LM, Allison KH, Allred DC, Bartlett JM, Bilous M, Fitzgibbons P, Hanna W, Jenkins RB, Mangu PB, Paik S, Perez EA, Press MF, Spears PA, Vance GH, Viale G, Hayes DF, American Society of Clinical O, College of American P (2013) Recommendations for human epidermal growth factor receptor 2 testing in breast cancer: American Society of Clinical Oncology/College of American Pathologists clinical practice guideline update. J Clin Oncol 31(31): 3997-4013.

Yarden Y, Sliwkowski MX (2001) Untangling the ErbB signalling network. Nat Rev Mol Cell Biol 2(2): 127-137.

This work is published under the standard license to publish agreement. After 12 months the work will become freely available and the license terms will switch to a Creative Commons AttributionNonCommercial-Share Alike 4.0 Unported License.

Supplementary Information accompanies this paper on British Journal of Cancer website (http://www.nature.com/bjc) 